\title{
Electrostriction of a near-critical fluid in microgravity
}

\author{
G. A. Zimmerli, ${ }^{1}$ R. A. Wilkinson, ${ }^{2}$ R. A. Ferrell, ${ }^{3}$ and M. R. Moldover ${ }^{4}$ \\ ${ }^{\prime}$ National Center for Microgravity Research, NASA Lewis Research Center, Cleveland, Ohio 44135 \\ ${ }^{2}$ Microgravity Science Division, NASA Lewis Research Center, Cleveland Ohio 44135 \\ ${ }^{3}$ Department of Physics, University of Maryland, College Park, Maryland 20742 \\ ${ }^{4}$ Physical and Chemical Properties Division, NIST, Gaithersburg, Maryland 20899
}

\begin{abstract}
We used interferometry to measure the electrostrictive increase of the density of sulfur hexaflouride $\left(\mathrm{SF}_{6}\right)$ near its critical point. The results at three temperatures $\left(T_{\mathrm{c}}+5.0 \mathrm{mK}, T_{\mathrm{c}}+\right.$ $10.0 \mathrm{mK}, T_{\mathrm{c}}+30.0 \mathrm{mK}$ with $\left.T_{\mathrm{c}}=319 \mathrm{~K}\right)$ agree with a calculation based on the Clausius-Mossotti relation and the restricted cubic model equation of state. To measure electrostriction, an inhomogeneous electric field $(\leq 26 \mathrm{kV} / \mathrm{cm})$ was applied to the $\mathrm{SF}_{6}$ sample by charging a fine wire that passed through it. These measurements were performed in microgravity so that the small electrostrictive density changes ( $\leq 3.5 \%$ in this work) would not be masked by the larger stratification of the fluid's density induced by the Earth's gravity. The predicted shifts of the critical temperature and density resulting from the electric field were too small to detect.
\end{abstract}




\section{INTRODUCTION}

Most experimental studies of electric field effects near critical points have been concerned with measurements of the dielectric anomaly ${ }^{1-5}$ (at low field strengths), and moderate field strength studies of binary liquid mixtures near consolute points ${ }^{6-8}$. Such mixtures are easily handled; however, they are not perfect insulators. Thus, applying an electric field inevitably leads to ohmic heating which, in turn, prevents the study of electric field effects in a mixture at equilibrium. In contrast, the present study is concerned with the single-component nonconducting fluid, sulfur hexafluoride $\left(\mathrm{SF}_{6}\right)$, near its liquid-vapor critical point. We measured the electric-field-induced increase of the density of $\mathrm{SF}_{6}$ in equilibrium at the temperatures $T_{c}+5.0$ $\mathrm{mK}, T_{\mathrm{c}}+10.0 \mathrm{mK}$, and $T_{\mathrm{c}}+30.0 \mathrm{mK}$, where $T_{c}$ is the critical temperature. The measured density increase was in quantitative agreement with theory.

The density increase was an example of "electrostriction," i.e. the deformation of a fluid or solid in the presence of an electric field. Near a critical point, electrostriction becomes more pronounced because of the diverging isothermal compressibility. The present work extends the electrostriction measurements of Hakim and Higham ${ }^{9}$ to highly-compressible near-critical fluids, where the compressibility is seven orders of magnitude higher than the liquids studied in reference 9. A preliminary analysis of our electrostriction data was reported in a previous publication, together with observations of the electric field-induced flows in the $\mathrm{SF}_{6}$ fluid in a microgravity environment ${ }^{4}$.

In the absence of free charges, and neglecting the effect of gravity, the force acting on a unit volume element of dielectric in an electric field of strength $E$ is ${ }^{11}$

$$
\mathbf{f}_{E}=-\frac{1}{2} \varepsilon_{0} E^{2} \nabla(\kappa)+\frac{1}{2} \varepsilon_{0} \nabla\left(E^{2} \rho d \kappa / d \rho\right)
$$

where $\varepsilon_{0}$ is the vacuum permittivity, $\kappa$ the dielectric constant, and $\rho$ is the density. For fluids, the dielectric constant is related to density through the Clausius-Mossotti relation, which states that the ratio $(\kappa-1) / \rho(\kappa+2)$ is a constant for any given dielectric. (Although the Clausius-Mossotti relation is only approximately valid for fluids, it is found to be sufficiently accurate for non-polar 
fluids at the density encountered near a critical point.) Thus, density gradients will be affected by the electric field, with regions of higher density being pulled into the regions of stronger electric field, leading to dielectrophoretic flow. The electric field force on a dielectric is usually small in comparison to gravity. However, in a microgravity environment, an electric field can be used to pump and confine liquids ${ }^{12}$ and it can induce convective flows ${ }^{13}$. Voronel and Giterman ${ }^{14}$ recognized the possibility of using strong electric fields to counter the gravity-induced stratification that occurs near the critical point as early as 1968 . Fields of tens of kilovolts/cm are required for samples that are a few millimeters high.

We are interested in solving for the density change in the fluid due to an applied electric field. In equilibrium, the electric volume force in the fluid is balanced by a pressure gradient such that $\mathbf{f}_{E}=\nabla p$. The electric volume force can be re-written as a single term,

$$
\nabla p=\frac{1}{2} \varepsilon_{0} \rho \nabla\left(E^{2} \frac{d \kappa}{d \rho}\right)
$$

Upon integrating, we find the desired relation between pressure and electric field:

$$
\int \frac{d p}{\rho}=\frac{1}{2} \varepsilon_{0} E^{2} \frac{d \kappa}{d \rho}
$$

For an incompressible fluid, we immediately find that the electrostrictive pressure is given by

$$
p_{E}=\frac{1}{2} \varepsilon_{0} E^{2} \rho \frac{d \kappa}{d \rho}=\varepsilon_{0} E^{2}(\kappa-1)(\kappa+2) / 6
$$

where we have used the Clausius-Mossotti relation, and $\kappa=1.28$ for $\mathrm{SF}_{6}$ at the critical density ${ }^{1,2}$. When a compressible fluid, such as $\mathrm{SF}_{6}$ near the critical point, is subjected to an electric field, there is an accompanying change of its density that can be calculated from Eq. (3) and the fluid's equation of state. In the limits of a linear response and isothermal conditions, the density change due to an electric field is 


$$
\frac{\Delta \rho}{\rho}=\beta_{T} p_{E}
$$

where $\beta_{\mathrm{T}}$ is the isothermal compressibility. Of course, the density change occurs only if the portion of the fluid subjected to the field is in contact with a sufficiently large reservoir of fluid outside the electric field.

Because of the relatively large compressibility encountered within a few millikelvin of a critical point, even a modest electric field produces an easily measured density change in a nonpolar fluid. For example, at $T_{\mathrm{c}}+5.0 \mathrm{mK}$ the isothermal compressibility of $\mathrm{SF}_{6}$ is $4.1 \times 10^{4} / P_{\mathrm{c}}$ where $P_{\mathrm{c}}$ is the critical pressure ( $3.76 \mathrm{MPa}$ ). An electric field of strength $10 \mathrm{kV} / \mathrm{cm}$ produces an electrostrictive pressure $p_{\mathrm{E}}=3.6 \times 10^{-7} P_{\mathrm{c}}$. The corresponding density increase is approximately $1.5 \%$, a change that was easily measured using interferometric techniques.

Earth-based measurements of the electrostriction effect near the liquid-vapor critical point are completely masked by gravity effects. The scale of gravity effects can be compared to electrostriction by noting that the gravitationally caused pressure gradient in $\mathrm{SF}_{6}$ at its critical

density $\left(730 \mathrm{~kg} / \mathrm{m}^{3}\right)$ is $2.9 \times 10^{-6} P_{\mathrm{c}}$ per millimeter of height. Thus, the gravitational pressure difference across an $\mathrm{SF}_{6}$ sample only $0.12 \mathrm{~mm}$ high equals the electrostrictive pressure produced by an electric field of $10 \mathrm{kV} / \mathrm{cm}$. Furthermore, in the Earth's gravity, stratification of the nearcritical $\mathrm{SF}_{6}$ leads to comparatively large dielectrophoretic forces due to the density gradient. Thus, applying an electric field to a stratified, near-critical fluid will cause flow. In this situation, it is virtually impossible to detect the more subtle electrostriction effect in the presence of the larger gravity-caused stratification and dielectrophoretic flow. By performing the experiment in a microgravity environment, we were able to avoid density stratification in the sample and thus make an accurate measurement of the electrostriction effect.

\section{EXPERIMENT APPARATUS}

The experiment was carried out using the Critical Point Facility ${ }^{15}(\mathrm{CPF})$ which flew on the Second International Microgravity Laboratory (IML-2) mission during the STS-65 Mission of the Space Shuttle Columbia during July 1994. The CPF was a multi-user instrument designed and built in Europe and operated by the European Space Agency. Five separate experiments were 
conducted sequentially in the CPF during the IML-2 mission. To start each experiment, mission specialists (astronauts) installed the experiment's unique sample cell unit (SCU) in the CPF. The CPF provided temperature control and it contained optical components, including light sources, shutters, and video and $35 \mathrm{~mm}$ cameras. As described in greater detail in another publication ${ }^{16}$, the $\mathrm{CPF}$ thermostat stabilized the temperature of the SCU so that temperature excursions were less than $0.1 \mathrm{mK}$ during intervals of many hours. The temperature gradients across the SCU were less than $100 \mu \mathrm{K} \mathrm{cm}^{-1}$.

The heart of the SCU used in this work was a coin-shaped volume of $\mathrm{SF}_{6}$ that was studied by interferometry. Schematic diagrams of our interferometer cell are shown in Figure 1. The $\mathrm{SF}_{6}$ was confined by two flat quartz windows and by a washer-shaped stainless-steel spacer. The inside diameter of the spacer was $12 \mathrm{~mm}$, and its thickness was $2 \mathrm{~mm}$. Approximately one quarter of this confined volume was occupied by a $1 \mathrm{~mm}$-thick, semicircular, quartz "button" attached to one window. The button divided the cell into two parts; the part near the fill tube had a $1 \mathrm{~mm}$ fluid thickness and the other part had a $2 \mathrm{~mm}$ fluid thickness.

The interferometer sample cell comprised one leg of a Twyman-Green interferometer. A multi-layer dielectric mirror coated the inner surface (in contact with the fluid) of one of the windows. Both surfaces of the other window had anti-reflection coatings. Interferograms were recorded once every six seconds by the CCD camera in CPF. The digital image was sent to the ground, recorded, and displayed. The 6-bit resolution of the CCD provided 64 gray levels and a spatial resolution of $35 \mu \mathrm{m}$ at the focal plane. Unfortunately, the interferometer optics were not focused at the sample cell. As described below, the effects of poor focus were accounted for when the images were compared with theory.

In addition to the optical coatings mentioned, both inner surfaces of the windows were coated with a conductive, transparent, indium-tin-oxide film. The conductive films made electrical contact to the spacer via the gold o-rings that were used to seal the cell. The films on the inner window surfaces and the spacer were kept at ground potential throughout the experiment.

A $0.125 \mathrm{~mm}$ diameter wire passed through the $2 \mathrm{~mm}$ thick section of the $\mathrm{SF}_{6}$ parallel to the edge of the quartz button and $1.8 \mathrm{~mm}$ away from its edge. The wire had been spot welded to 
electrical feedthroughs, which, in turn, were epoxied into the spacer. The wire was charged to $500 \mathrm{~V}$ to apply an inhomogeneous electric field to the sample.

As described in Reference 16, the sample cell was filled to within $0.3 \%$ of the critical density with $99.999 \%$ pure $\mathrm{SF}_{6}$.

During the experiment, commands were sent from the ground to CPF to turn the voltage on and off, to take photographic and CCD images of the interferograms, and to change the set-point temperature of the thermostat.

Seventy five minutes after starting the experiment, CPF brought the SCU to $T_{\mathrm{c}}+1 \mathrm{~K}$ and maintained it there for 30 minutes. During this interval, the $\mathrm{SF}_{6}$ equilibrated, attaining a uniform temperature and density. Then the SCU was taken through $T_{\mathrm{c}}$ using a sequence of ramps designed to minimize the density inhomogeneities in the $\mathrm{SF}_{6}$. First, the temperature was ramped down to $T_{\mathrm{c}}+30 \mathrm{mK}$ in 20 minutes and it was held there for 5 minutes. Then, the temperature was ramped down to $T_{c}+3 \mathrm{mK}$ in 40 minutes and held there for 10 minutes. Finally, the temperature was ramped through $T_{\mathrm{c}}$ at the rate $-5 \times 10^{-7} \mathrm{~K} / \mathrm{s}$ while the video images acquired every six seconds were recorded. A dramatic increase in turbidity throughout the $\mathrm{SF}_{6}$ sample was bracketed by two images. This was identified as the onset of spinodal decomposition and it was assumed to define $T_{\mathrm{c}}$ with an uncertainty of less than $0.1 \mathrm{mK}$ relative to the CPF thermometers. (All uncertainties stated are a single standard uncertainty, i.e. the coverage factor $k=1$.) The microgravity value of $T_{\mathrm{c}}$ agreed with the value determined on Earth prior to this measurement to within the latter's larger uncertainty, $0.3 \mathrm{mK}$. All subsequent temperature measurements were made relative to the microgravity value of $T_{\mathrm{c}}$ with uncertainties that were less than $0.1 \mathrm{mK}$.

\section{ELECTROSTRICTION FRINGE PATTERNS}

Changes in the fluid density distribution were measured by monitoring changes in the interferometric fringe pattern, which sensed changes in the optical path length. The nominal optical path length of the laser light in the fluid was $N \lambda=2 \ln$. Here, $N$ is the number of wavelengths required to pass twice through the fluid, $\lambda=633 \mathrm{~nm}$ was the wavelength of the light in vacuum, $l=2 \mathrm{~mm}$ was the thickness of the fluid, and $n=1.092$ is the index of refraction 
of the $\mathrm{SF}_{6}$ at the critical density ${ }^{17}$. When the density of the $\mathrm{SF}_{6}$ changed, the optical path length changed. The resulting fringe shift, or change in fringe order, was

$$
\Delta N=(2 l / \lambda)(\delta \rho / \rho) n^{\prime}
$$

where $\delta \rho$ was the density change and

$$
n^{\prime}=\rho d n / d \rho=\left(n^{2}-1\right)\left(n^{2}+2\right) / 6 n
$$

is obtained from the Lorentz-Lorenz relation connecting the density to index of refraction. Thus, a relative density change of $0.01 \%$ in the $2 \mathrm{~mm}$ thick section of our fluid sample would produce a fringe shift of $\Delta N=0.056$ fringes, which was approximately twice the limit of our resolution.

The fringe pattern displayed in Figure 2(a) was obtained under conditions where the density was homogeneous $\left(T_{c}+1 \mathrm{~K}\right.$ with the electric field off). This fringe pattern was determined by the tilt of the reference mirror of the interferometer together with distortions resulting from the optics. The bending of the fringes around the edges of the cell is due to bowing in the quartz windows, which results from the forces needed to seal the cell. A discontinuity in the fringe pattern is visible across the diameter of the cell. This resulted from the semi-circular quartz button, which changes the optical path length relative to the other half of the cell.

Figure 2(b-d) shows the equilibrium fringe patterns when the wire was charged to a potential of $500 \mathrm{~V}$ at $T_{\mathrm{c}}+30.0 \mathrm{mK}, T_{\mathrm{c}}+10.0 \mathrm{mK}$, and $T_{\mathrm{c}}+5.0 \mathrm{mK}$, respectively. The fringes near the wire were bent by the density increase near the wire due to electrostriction. Upon applying the electric field, we did not immediately observe the density change given by Eq. (5). The full density change density given by Eq. (5) is an isothermal calculation. Turning on the electric field heats the fluid a small amount due to the electrocaloric effect ${ }^{18}$. The excess heat gradually diffuses out of the fluid, the density change gradually builds up, and the fluid returns to the original temperature after approximately a thermal diffusion time constant $\tau=l^{2} / \pi^{2} D_{t}$. Because the thermal diffusivity of the fluid $D_{t}$ approaches zero as the critical point is approached, it is necessary to wait increasingly longer times as the temperature approaches $T_{\mathrm{c}}$. The fringe patterns shown in Figs. 2(b-d) were obtained after leaving the $500 \mathrm{~V}$ potential on for 3, 6, and 14 
hours respectively. The immediate, or adiabatic, density change is predicted by substituting the adiabatic compressibility $\beta_{\mathrm{S}}=\left(C_{\mathrm{p}} / C_{\mathrm{v}}\right)^{-1} \beta_{\mathrm{r}}$ for the isothermal compressibility in Eq. (5). The adiabatic density change is much smaller than the isothermal change because $C_{\mathrm{p}} / C_{\mathrm{v}}$, the ratio of the specific heat at constant pressure to that at constant volume, diverges strongly near the critical point. Indeed, the adiabatic density change was too small for us to detect close to $T_{c}$; however, the isothermal density change was easily measured.

\section{THEORY}

The electric field produced by a charged wire passing halfway between two grounded parallel plates separated by a distance $l$ can be calculated by solving Laplace's equation with appropriate boundary conditions. (This model for the cell neglects the small, position-dependent variations of the dielectric constant resulting from electrostriction.) Choosing the coordinate system such that the $\mathrm{x}$-axis is perpendicular to the wire and parallel to the grounded window surfaces, and the z-axis is perpendicular to the windows, the result for the square of the electric field is

$$
E^{2}=\frac{C^{2} V^{2}}{2 l^{2} \varepsilon_{0}^{2}}\left(\cosh \left(\frac{2 \pi x}{l}\right)-\cos \left(\frac{2 \pi z}{l}\right)\right)^{-1}
$$

where $V$ is the voltage applied to the wire, and $C$, the capacitance per unit length of the wire of radius $a$, is given by $C=2 \pi \varepsilon_{0}[\ln (2 l / \pi a)]^{-1}$. In our sample cell geometry, the electric field produced at the surface of the wire by a $500 \mathrm{~V}$ potential is $26 \mathrm{kV} / \mathrm{cm}$. The electric field falls off rapidly with distance because of the screening by the grounded windows (an essential feature of the cell fabrication), and becomes $1 \mathrm{kV} / \mathrm{cm}$ at $x=1 \mathrm{~mm}$ from the wire. The effect of the stepped window geometry on the electric field was evaluated using a Schwartz-Christoffel transformation ${ }^{19}$. Because the field is weak near the step edge and the electrostriction effect is proportional to $E^{2}$, the effect of the step edge on our results is negligible. 
With the wire charged to a $500 \mathrm{~V}$ potential, the electrostrictive pressure $p_{E}$ at the surface of the wire is $2.4 \times 10^{-6} P_{\mathrm{c}}$. Although this is a relatively small pressure increase, the compressibility is large enough near the critical point such that the electrostrictive pressure induces a nonlinear response in the compressibility. In the linear regime, the isothermal compressibility $\beta_{\mathrm{T}}=(1 / \rho)(\partial \rho / \partial P)_{\mathrm{T}}$ diverges on the critical isochore near the critical point as $\beta_{\mathrm{T}}=\Gamma t^{-1.24} / P_{\mathrm{c}}$ where $t=\left(T-T_{\mathrm{c}}\right) / T_{\mathrm{c}}$ is the reduced temperature, and $\Gamma=0.0459$ for $\mathrm{SF}_{6}$ in the restricted cubic mode $^{20}$. Close to the wire, and within about $20 \mathrm{mK}$ of the critical temperature, Eq. (5) is no longer a good representation of the density change, because $\beta_{\mathrm{T}}$ can not be approximated by a temperature-dependent constant, i.e. independent of $p_{\mathrm{E}}$. Thus, it is necessary to use Eq. (3) together with the equation of state of $\mathrm{SF}_{6}$ to numerically compute the density increase due to the electrostrictive pressure. Figure 3 shows the density change as a function of pressure, normalized to values at the critical point, as calculated from the restricted cubic model parameters found in Ref. 20.

The change in optical path length of a ray passing through the fluid is calculated by integrating the density increase along the light path and using the Lorentz-Lorenz relation to relate the change in fluid density to a change in index of refraction. The change in fringe order at a lateral distance $x$ from the wire is then given by

$$
\Delta N(x)=\frac{2}{\lambda} n^{\prime} \int_{-l / 2}^{l / 2} \frac{\Delta \rho(x, z)}{\rho} d z
$$

In the linear regime, the compressibility is independent of position and the integral can be evaluated analytically. The result is

$$
\Delta N(x)=\frac{n^{\prime} \beta_{T}}{12 \lambda h \varepsilon_{0}}(\kappa-1)(\kappa+2) c^{2} V^{2} \operatorname{csch}(2 \pi x / l) .
$$

The predictions of Eq. (9) and Eq. (10) agree very well at $T_{\mathrm{c}}+30.0 \mathrm{mK}$ because the pressure and density are in the linear regime. However, at temperatures close to $T_{\mathrm{c}}$ and close to the wire, it is necessary to use the general form given by Eq. (9), and compute the integral numerically. To 
evaluate Eq. (9), we first calculate the right hand side of Eq. (3) at a given location $(x, z)$. The result is compared with the numerical computation of the integral of $\mathrm{d} p / \rho$ at the temperature of interest, which gives a matching result at some value of $\Delta \rho$. This value of $\Delta \rho$ is then used in evaluating Eq. (9). Figure 4 shows the predicted fringe shift as a function of distance from the wire, using both the analytic expression of Eq. (10) and the numerical analysis based on Eqs. (3) and (9). As expected, the two results agree when the fluid responds linearly to the electrostrictive pressure.

Finally, we must consider the possibility of a shift in the critical temperature $\Delta T_{\mathrm{c}}(E)$ and density $\Delta \rho_{\mathrm{c}}(E)$ due to the electric field. Landau and Lifshitz ${ }^{18}$ show that an electric field changes the free energy of the fluid, resulting in a shift of the critical point. In Appendix A, we evaluate the shifts in the critical temperature and the critical density near the surface of the wire, where the electric field is greatest. The results are $\Delta T_{\mathrm{c}}(26 \mathrm{kV} / \mathrm{cm})=0.2 \mathrm{mK}$ and $\Delta \rho_{\mathrm{c}}(26 \mathrm{kV} / \mathrm{cm})=$ $6.9 \times 10^{5} \rho_{\mathrm{c}}$. The very small shift in the critical density could not be detected in this experiment; the small shift in the critical temperature might have been barely detectable if it were not confined to a very small volume in the immediate vicinity of the wire. The interference patterns that we analyzed resulted from optical paths that integrated the density through the full thickness of the cell. Thus, the effects of the electric field on the critical point were too small to influence the present experiment.

\section{DATA ANALYSIS}

To analyze the fringe patterns, the original digital images were rotated using commercial software so that the wire was parallel to pixel columns. A subset of the original images, centered on the wire and occupying a region approximately $3 \mathrm{~mm}$ wide ( 84 pixels) by $7 \mathrm{~mm}$ tall (175 pixels), was used to analyze the fringe shift. An identical section from each of the interferograms shown in Fig. 2 was used in the analysis. The task is to measure the fringe shift $\Delta N(x)$ as a function of distance from the wire. Because of the periodic nature of the fringe pattern and the symmetry of the problem, the image analysis is well suited to Fourier analysis.

The subset of each image is represented by a matrix of intensity data, $I(x, y)$. Each column of pixels, parallel to the wire and at position $x_{0}$, is thus represented by an array $I\left(x_{0}, y\right)$. We then 
take the Fourier transform of $I\left(x_{0}, y\right)$, which yields magnitude and phase data at a range of spatial frequencies. Because the intensity pattern is nearly periodic along a column of pixels, the magnitude data exhibit a strong peak at the spatial frequency of the fringe pattern. The phase data at that frequency of interest determines the phase of the fringe pattern to within an arbitrary constant. The process is repeated for each of the 84 columns of intensity data, and again for each temperature of interest. A density increase near the wire due to electrostriction produces a change in the phase of the fringe pattern, but the spatial frequency of the fringe pattern parallel to the wire remains the same.

Figure 5 shows phase data at $T_{\mathrm{c}}+1 \mathrm{~K}$ and $T_{\mathrm{c}}+10.0 \mathrm{mK}$ that were computed from a Fourier analysis of images shown in Fig. 2. The Fourier analysis accurately reproduces the peak in the phase shift near pixel 43 where the wire was imaged. However, far from the wire, where the electric field and the electrostrictive density changes were always small, the phases for the two temperatures plotted in Fig. 5 do not coincide. Thus, the background fringe pattern upon which the peak is superimposed appears to have temperature dependence. An analysis of other images at $T_{\mathrm{c}}+1 \mathrm{~K}$ and $T_{\mathrm{c}}+3 \mathrm{~K}$, spanning an 80 hour period, indicated that the background fringe pattern was changing slowly with time and had a slight temperature dependence. Such a change in the background fringe pattern might have been caused by creeping motion of one or more of the optical components within CPF. If the sample cell had leaked, there would have been a drift in the background fringe pattern in the direction opposite to that which was observed.

We expected the fringe shift due to electrostriction to be symmetrical on both sides of the wire. However, the typical fringe pattern had a small $(<0.2$ fringe) asymmetry. The asymmetry was consistent with a slight rotation of the background fringe pattern and it was detected even far from $T_{\mathrm{c}}$, for example, upon comparing the phase data at $T_{\mathrm{c}}+1 \mathrm{~K}$ and $T_{\mathrm{c}}+3 \mathrm{~K}$. The rotation of the fringe pattern was probably caused by movement of the same optical component that produced the translation of the background fringe pattern displayed in Fig. 5.

The final data analysis eliminated the effect of the rotation by averaging data from the two sides of the wire. The analysis also compensated for the time-dependent phase drift of the fringe patterns by subtracting the phase data at $T_{\mathrm{c}}+1 \mathrm{~K}$ from all the other sets of phase data and adding a constant to the results, where the constant was chosen to yield zero phase shift far from the wire. 
Because the CPF interferometer optics were not focused at the sample cell, we found that it was also necessary to account for the effect of refraction ${ }^{21}$. A ray exiting the fluid in the vicinity of the wire is bent away from the normal due to refraction in the fluid, as shown schematically in Fig. 6. The interference pattern recorded by the CCD camera is thus dependent on the position of the imaging plane. If the camera were focused on the sample cell, no correction would be necessary. Using detailed data about the interferometer optical train ${ }^{22}$ we calculated the location of the imaging plane. We find that the distance from the sample cell to the imaging plane is $z_{0}=6.6 \mathrm{~cm}$.

The effect of refraction on the recorded interferograms is twofold. First, a refracted ray is displaced from its "true" position, i.e. the position of interest where it exits the sample cell. The refracted ray has the effect of carrying phase information from, for example, the lateral coordinate $x_{0}<0$ where it exits the sample cell to a new position $x_{0}{ }^{\prime}=x_{0}+z_{0} \tan \theta$ at the imaging plane, where $\theta$ is the angle the refracted ray subtends with the normal. For example, a ray passing close to the left side of the wire can be refracted to the right side of the wire at the imaging plane. In fact, a close inspection of the interferograms near $T_{\mathrm{c}}$ in Fig. 2 reveals a faint extension of the fringe pattern from one side of the wire to the other side. The subtle effect is more readily observed when the interferogram is viewed at a grazing angle. A large magnified image of the interferograms was used to manually trace the extended fringe pattern, which originated from the opposite side of the wire. Data from manually traced fringes on both sides of the wire were averaged together to eliminate the effect of rotation in the background fringe pattern, as discussed previously. Fringe shift data from the manually traced fringes was appended to the Fourier transform data, and the two methods of analysis were found to yield a smooth match of the data in the vicinity of the wire. To summarize, the fringes start on one side of the wire and continue on the opposite side of the wire as a faint extension because of refraction. The Fourier analysis was used on the originating side; the hand-traced fringe analysis was used on the faint extensions.

A second consequence of refraction is the addition of a path-length error. A ray that exits the fluid at angle $\theta$ travels an extra distance relative to a ray that is not refracted. The additional path-length traveled by the refracted ray increases the phase from the true value by the amount $z_{0} \pi \theta^{2} / \lambda$. The angle $\theta$ the refracted ray subtends with the normal is calculated from the expression 
$\sin \theta=(\lambda / 2 \pi)(d \varphi / d x)$ where $\varphi$ is the phase of the wavefront at the plane $z_{0}=0$ where the wavefront exits the fluid. The phase of the wavefront is calculated from Eq. 9, noting that a change in fringe order $\Delta N=1$ corresponds to a phase change $\Delta \varphi=2 \pi$.

Figure 7 shows a comparison between theory and the measured fringe shift due to electrostriction, as a function of distance from the wire. Data points at $5.0 \mathrm{mK}, 10.0 \mathrm{mK}$, and $30.0 \mathrm{mK}$ above $T_{\mathrm{c}}$ are from an analysis of the interferograms in Fig. 2. The solid line is the theoretical fringe shift, which accounts for the non-linearity in the compressibility and the effect of refraction. The agreement between theory and experiment is excellent; the differences are within the noise of the fringe resolution which is of order 0.03 fringes in the vicinity of the wire. At $T_{\mathrm{c}}+5.0 \mathrm{mK}$, the fluid density ranges from $\rho_{\mathrm{c}}$ far from the wire, to $1.035 \rho_{\mathrm{c}}$ close to the wire due to the electrostriction effect. Data points for $x>0$ are from the Fourier transform analysis of the fringe patterns, and $x<0$ data is from the analysis of hand-traced fringes. The only adjustable parameter used in the data analysis is the small phase shift added to the Fourier transform data to force the fringe shift to zero far from the wire. As discussed previously, this offset is independent of position and is due to a small drift in the background fringe pattern.

The dashed line in Fig. 7 is the fringe shift calculated from Eq. (9), which accounts for the non-linearity in the compressibility but does not include the effect of refraction. In effect, refraction causes a one-to-one mapping of points in the dashed line to points in the solid line. By comparison, we find that refraction has a major influence on the fringe pattern close to the wire. As predicted, rays that exit the fluid close to wire are refracted to the other side of the wire at the imaging plane. Once again, we point out that accounting for refraction would not be necessary if the optics were focused on the sample cell. 
DRAFT $11 / 18 / 98$

\section{CONCLUSION}

We have measured the electrostriction effect surrounding a charged wire in a near-critical sample of $\mathrm{SF}_{6}$, at temperatures $5.0 \mathrm{mK}, 10.0 \mathrm{mK}$, and $30.0 \mathrm{mK}$ above $T_{\mathrm{c}}$. As theory predicts, the electrostriction effect diverges as the compressibility diverges near the critical point. By performing the experiment in a microgravity environment, we were able to avoid gravitational stratification of the fluid density. The electrostrictive density increase was measured by analyzing interferometric fringe patterns from the fluid sample. Because of the low thermal diffusivity of the fluid, the density increase is slow to develop. In the limit of a linear response and neglecting the effect of refraction, the fringe shift can be solved analytically and is given by Eq. (10). Closer to the critical point, and near the wire where the electric field is high, it is necessary to account

for the nonlinear response of the fluid compressibility by numerically evaluating the equation of state and computing the integrals in Eq's. (3) and (9). Using the restricted cubic model equation of state for $\mathrm{SF}_{6}$ and accounting for the effect of refraction on the out-of-focus interferometric images, we obtain quantitative agreement between electrostriction theory and data obtained in a microgravity environment.

\section{ACKNOWLEDGEMENTS}

We would like to thank R. Gammon, H. Hao, R. Kusner, W. Johnson, M. Bayda, H. Nahra and the entire CPF team for participating in the experiment operations. We are grateful to NASA, ESA, and the STS-65 crew, all whom made this experiment possible. We also thank K.Y. Min and J. Bahattacharjee for useful discussions. This work was supported by NASA through the Microgravity Science and Applications Division.

\section{APPENDIX A: SHIFT OF THE CRITICAL POINT DUE TO AN ELECTRIC FIELD}

We estimate the effect of an electric field on the critical temperature and critical density and show that it had a negligible effect on the present study of the electrostriction effect near the critical point. We follow Landau and Lifshitz ${ }^{18}$ who considered the free energy of a dielectric in the presence of an electric field and showed that the chemical potential of the fluid is 


$$
\mu=\mu_{0}-\frac{1}{2} \varepsilon_{0} E^{2}\left(\frac{\partial \kappa}{\partial \rho}\right)_{T}
$$

where $\mu_{0}$ is the chemical potential in the absence of an electric field. The conditions for the existence and stability of the critical point become

$$
\begin{aligned}
& \left(\frac{\partial \mu}{\partial \rho}\right)_{E, T}=\frac{\partial \mu_{0}}{\partial \rho}-\frac{1}{2} \varepsilon_{0} E^{2}\left(\frac{\partial^{2} \kappa}{\partial \rho^{2}}\right)=0 \\
& \left(\frac{\partial^{2} \mu}{\partial \rho^{2}}\right)_{E, T}=\frac{\partial^{2} \mu_{0}}{\partial \rho^{2}}-\frac{1}{2} \varepsilon_{0} E^{2}\left(\frac{\partial^{3} \kappa}{\partial \rho^{3}}\right)=0 .
\end{aligned}
$$

In an earlier edition of their book, Landau and Lifshitz ${ }^{18}$ calculated the shift of critical temperature $\Delta T_{c}$ by considering a Taylor series expansion of $(\partial P / \partial \rho)_{T}$ around the critical point. However, their result contains the factor $\left(\partial^{2} P / \partial \rho \partial T\right)_{T_{c}, \rho_{c}}$, which goes to zero at the critical point. The corresponding expression for the shift in critical density using a similar analysis ${ }^{14}$ also contains terms that go to zero at the critical point.

We have evaluated the shift of critical point by computing the thermodynamic derivatives in Eqs. (A2) and (A3) using the restricted cubic model equation of state. The derivative $\partial \mu_{0} / \partial \rho$ is inversely proportional to the isothermal compressibility, and is to be evaluated at the new critical point $T_{c}(E), \rho_{c}(E)$. Assuming the shift in critical density is negligibly small (which is verified below), Eq. (A2) gives the shift in critical temperature as

$$
\Delta T_{c}=\left(\frac{1}{2} \Gamma \varepsilon_{0} E^{2} \frac{\partial^{2} \kappa}{\partial \rho^{2}}\right)^{\frac{1}{\gamma}} T_{c}
$$

where $\gamma=1.24, \Gamma=(k / a)\left(\rho_{c}{ }^{2} / P_{c}\right)$ is the amplitude of a symmetrized isothermal compressibility, and, for $\mathrm{SF}_{6}, k=1.01$ and $a=22.0$ are the two, system-dependent scale factors that appear in the 
restricted cubic model. In the mean-field theory, $\gamma=1$, and Eq. (A4) reduces to the expression found by Onuki ${ }^{23}$.

The shift in the critical density can be calculated from Eq. (A3). Using the restricted cubic model expressions found in reference 20 , the condition specified by Eq. (A3) becomes

$$
\frac{2 a P_{c} r^{\gamma}}{k \rho_{c}^{3}}\left[\frac{\gamma b^{2} \theta}{k q(\theta)} r^{-\beta}-1\right]=\frac{1}{2} \varepsilon_{0} E^{2} \frac{\partial^{3} \kappa}{\partial \rho^{3}}
$$

and it is to be evaluated at the new critical temperature and density. The parametric variables $r$ and $\theta$ are the parametric variables used to define the temperature and density in the restricted cubic model coupled equations, and $b^{2}$ and $q(\theta)$ are also defined in the model and can be found in reference 12. To solve Eq. (A5) for the shift in critical density, we first note that the reduced temperature is given by $\Delta T_{C} / T_{c}$. Then, the value of the parameter $\theta$ is found which satisfies Eq. (A5). Once $r$ and $\theta$ are known, the shift in critical density $\Delta \rho_{c} / \rho_{c}$ is easily calculated.

We evaluated the derivatives $\left(\partial^{\mathrm{n}} \kappa \partial \rho^{\mathrm{n}}\right)_{\mathrm{T}}$ using the Clausius-Mossotti relation, which states that the ratio $(\kappa-1) / \rho(\kappa+2)$ is a constant for any given dielectric. Thus, taking $\rho \propto(\kappa-1) /(\kappa+2)$ we find

$$
\rho^{2} \frac{\partial^{2} \kappa}{\partial \rho^{2}}=\frac{2}{9}(\kappa-1)^{2}(\kappa+2)
$$

and

$$
\rho^{3} \frac{\partial^{3} \kappa}{\partial \rho^{3}}=\frac{2}{9}(\kappa-1)^{3}(\kappa+2) .
$$

When charged to a $500 \mathrm{~V}$ potential, the electric field at the surface of the wire is $26 \mathrm{kV} / \mathrm{cm}$ and the corresponding shift in critical temperature of $\mathrm{SF}_{6}$ calculated from Eq. (A4) is $\Delta T_{c}=0.2 \mathrm{mK}$. This shift is small in comparison with our closest approach to $T_{c}$; namely, $T_{c}+5.0 \mathrm{mK}$ and it had a negligible effect on our results. We also note that the shift decreased rapidly with the distance from the wire. 
At $500 \mathrm{~V}$, the shift in critical density calculated from Eq. (A5) is $\Delta \rho=6.9 \times 10^{-5} \rho_{\mathrm{c}}$ at the wire. This was far too small for us to detect. 


\section{References}

1. R. Hocken, M. A. Horowitz, and S. C. Greer, Phys. Rev. Lett. 37, 964 (1976).

2. B. J. Thijsse, J. Chem. Phys. 74, 4678 (1981).

3. M. W. Pestak and M. H. W. Chan, Phys. Rev. Lett. 46, 943 (1981).

4. J. L. Tveekrem, S. C. Greer, and D. T. Jacobs, Macromolecules 21, 147 (1988).

5. J. Hamelin, B. R. Gopal, T. K. Bose, and J. Thoen, Phys. Rev. Lett. 74, 2733 (1995).

6. P. Debye and Kleboth, J. Chem. Phys. 42, 3155 (1965).

7. Y. Yosida, Phys. Lett. 65A, 161 (1978).

8. D. Wirtz and G. G. Fuller, Phys. Rev. Lett. 71, 2236 (1993).

9. S. S. Hakim and J. B. Higham, Proc. Phys. Soc. 80, 190 (1962).

10. G. Zimmerli, R. A. Wilkinson, R. A. Ferrell, H. Hao and M. R. Moldover, Proc. of the 30th 1995 National Heat Transfer Conference - Volume 3, ASME, p. 121 (1995).

11. J.A. Stratton, Electromagnetic Theory, Chap. 2 (McGraw-Hill, New York, 1941).

12. J. R. Melcher and M. J. Hurwitz, J. Spacecraft and Rockets 4, 864 (1967).

13. J. E. Hart, G. A. Glatzmaier, and J. Toomre, J. Fluid Mech. 173, 519 (1986).

14. A. V. Voronel and M. Sh. Giterman, Sov. Phys. JETP 28, 1306 (1969).

15. M. Cork, T. Dewandre, and D. Hueser, ESA Bulletin 74, 36 (1993).

16. R. A. Wilkinson, G. Zimmerli, H. Hao, M. R. Moldover, R. F. Berg, W.L. Johnson, R. A. Ferrell and R. W. Gammon, Phys. Rev. E 57, 436 (1998).

17. D. S. Cannell, Phys. Rev. A 15, 2053 (1977).

18. L. D. Landau and E. M. Lifshitz, Electrodynamics of Continuous Media, p.83 (Pergamon Press, 1960).

19. V. Kostur, private communication

20. M.R. Moldover, J.V. Sengers, R.W. Gammon, and R.J. Hocken, Rev. Mod. Phys. 51, 79 (1979).

21. C. M. Vest, Holographic Interferometry, (Wiley, New York, 1979).

22. T. Dewandre kindly provided data regarding the CPF optics.

23. A. Onuki, Europhys. Lett. 29, 611 (1995). 


\section{Figure Captions}

FIG. 1. Schematic diagrams of the sample cell. The fluid-filled volume is $12 \mathrm{~mm}$ in diameter. Half of this region is $2 \mathrm{~mm}$ thick; the other half is $1 \mathrm{~mm}$ thick. A fine wire passing through the fluid was charged to $500 \mathrm{~V}$ to generate the electric field.

FIG 2. Interferograms of the $\mathrm{SF}_{6}$ fluid in a low gravity environment. The bending of the fringes near the wire signifies the density increase due to the electrostriction effect. (a) $T_{\mathrm{c}}+1 \mathrm{~K}$ with no electric field. (b) $T_{\mathrm{c}}+30.0 \mathrm{mK}$ after $3 \mathrm{hr}$ at $500 \mathrm{~V}$. (c) $T_{\mathrm{c}}+10.0 \mathrm{mK}$ after $6 \mathrm{hr}$ at $500 \mathrm{~V}$. (d) $T_{\mathrm{c}}+5.0 \mathrm{mK}$ after $14 \mathrm{hr}$ at $500 \mathrm{~V}$.

FIG 3. The relative density as a function of the relative pressure of $\mathrm{SF}_{6}$. The calculated curves used the restricted cubic model. When the wire was charged to $500 \mathrm{~V}$, the electrostrictive pressure at the surface of the wire was $2.4 \times 10^{-6} P_{c}$, as indicated by the dashed line.

FIG. 4. Predicted electrostrictive fringe shifts as a function of distance from the center of the wire. The dashed curves resulted from using the linear approximation to the equation of state Eq. (10). The solid curves are the more accurate, numerical results using the restricted cubic model equation of state and Eqs. (3) and (9).

FIG. 5. Phase data at $T_{\mathrm{c}}+1.0 \mathrm{~K}$ and $T_{\mathrm{c}}+10.0 \mathrm{mK}$ from a Fourier analysis of the interferograms shown in Figure 2.

FIG. 6. Schematic showing the effect of refraction. Rays passing close to the wire are refracted to the other side of the wire at the image plane $\sigma$. In addition to being displaced from its original $x$ coordinate, the refracted ray travels an extra distance, thus producing an extra phase shift at the imaging plane.

FIG 7. Measured electrostriction fringe shift in a $\mathrm{SF}_{6}$ sample as a function of distance $x$ from the center of the wire: $T_{\mathrm{c}}+5.0 \mathrm{mK}(0) ; T_{\mathrm{c}}+10.0 \mathrm{mK}(\nabla)$; and $T_{\mathrm{c}}+30.0 \mathrm{mK}(\square)$. The solid lines 
are the theoretical fringe shifts including the nonlinear response of the fluid and the effects of refraction. The dashed curves include nonlinearity but neglect the effects of refraction. 
Flanges
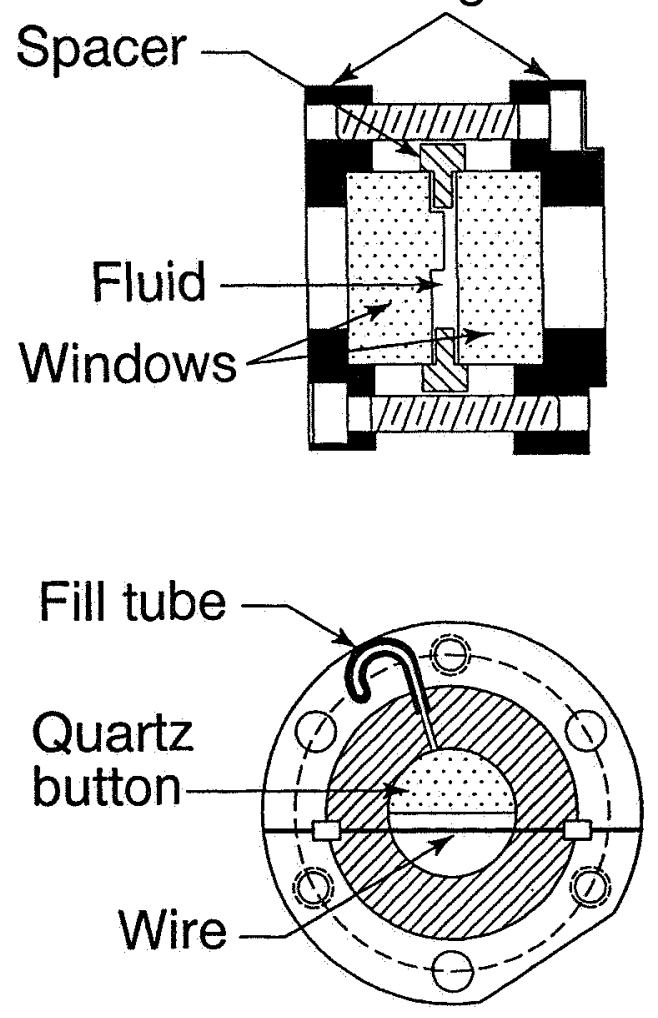

Figure 1 


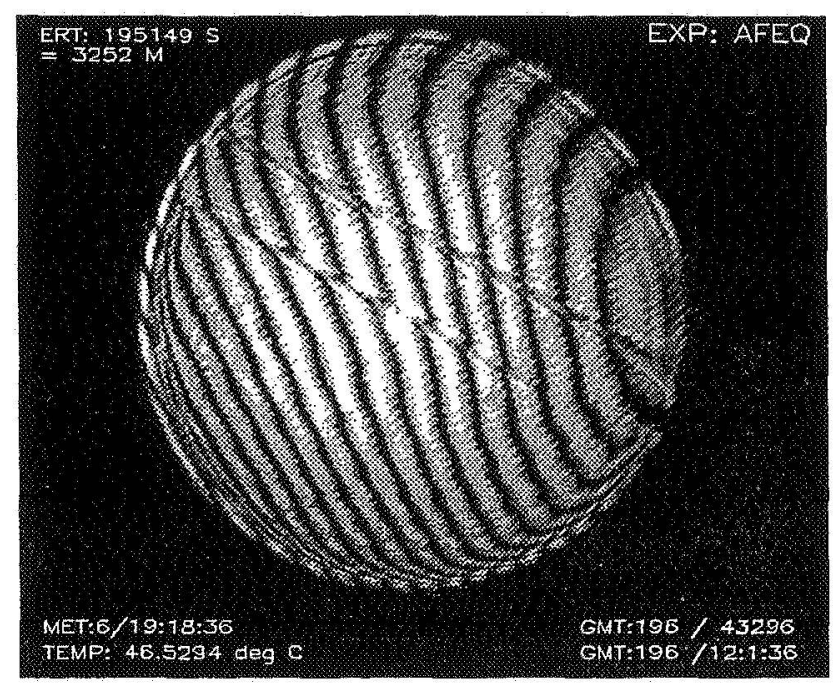

Figure 2a

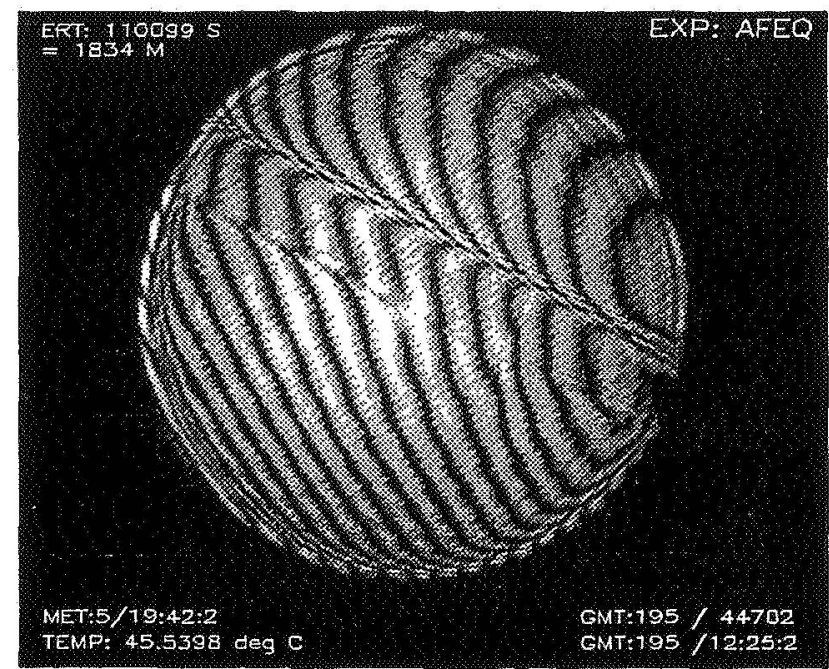

Figure 2c

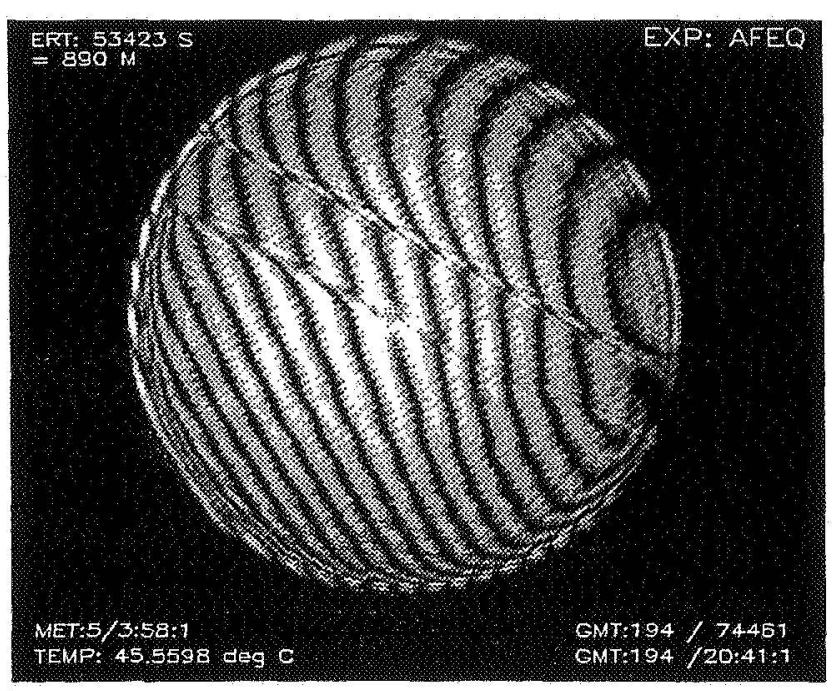

Figure $2 b$

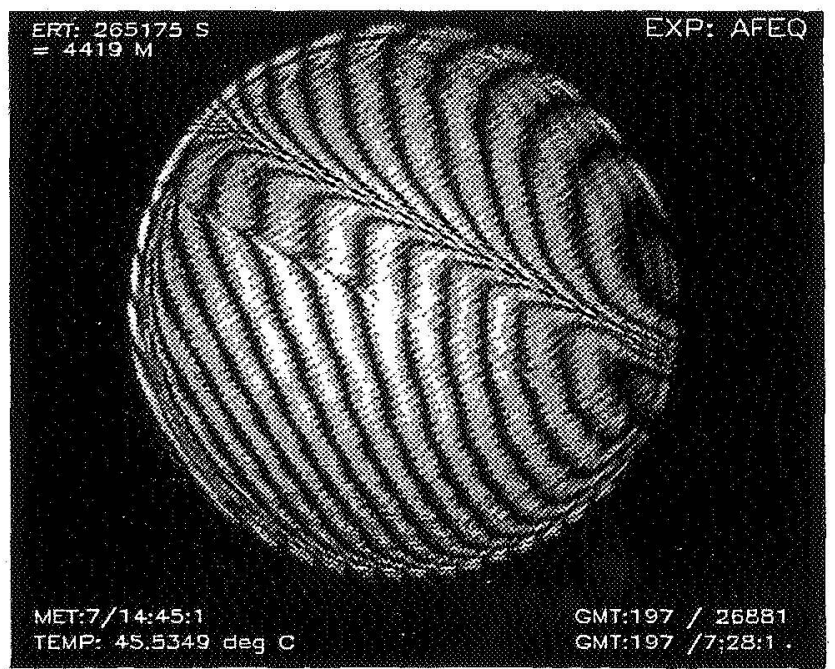

Figure 2d 


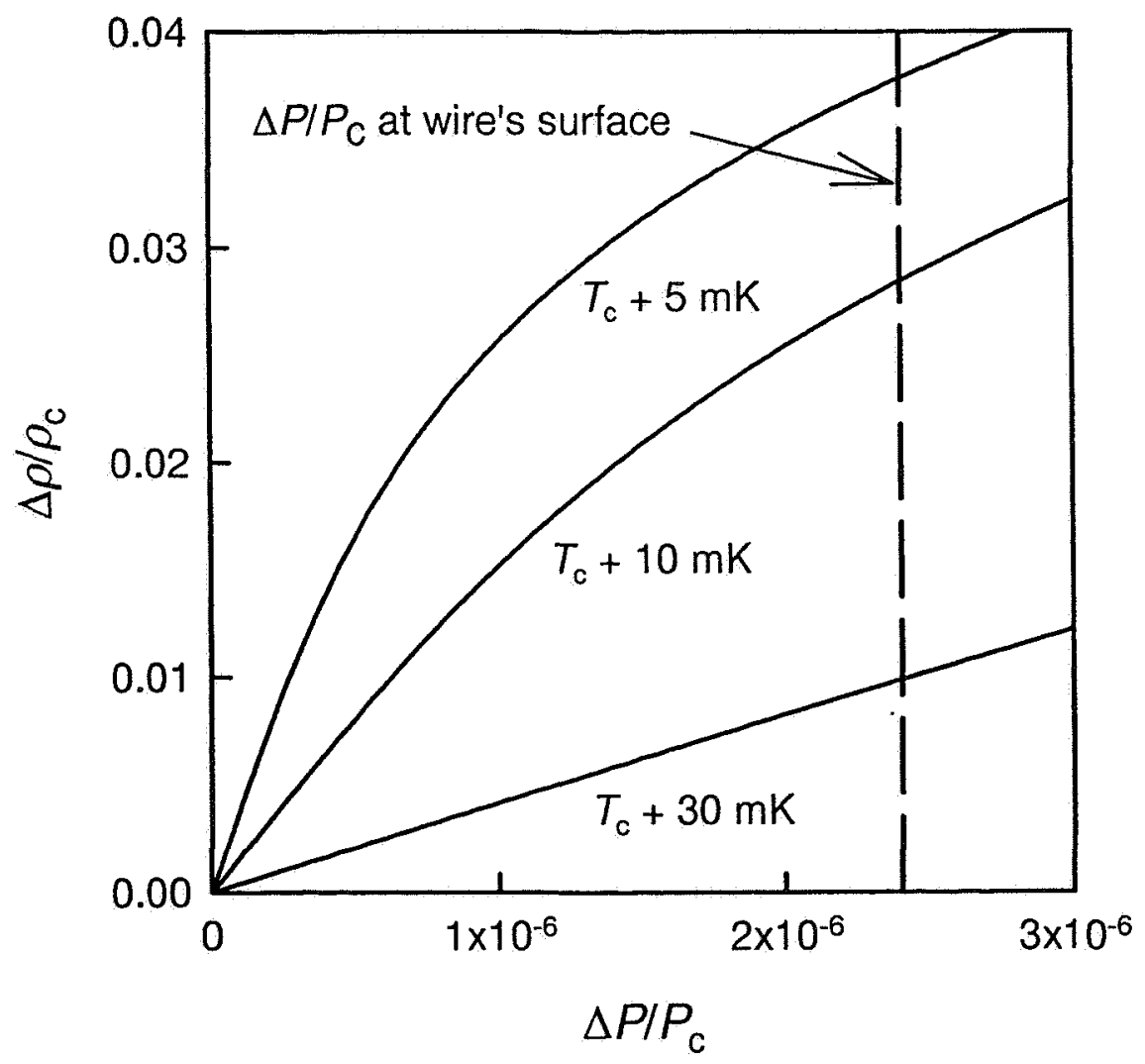

Figure 3 


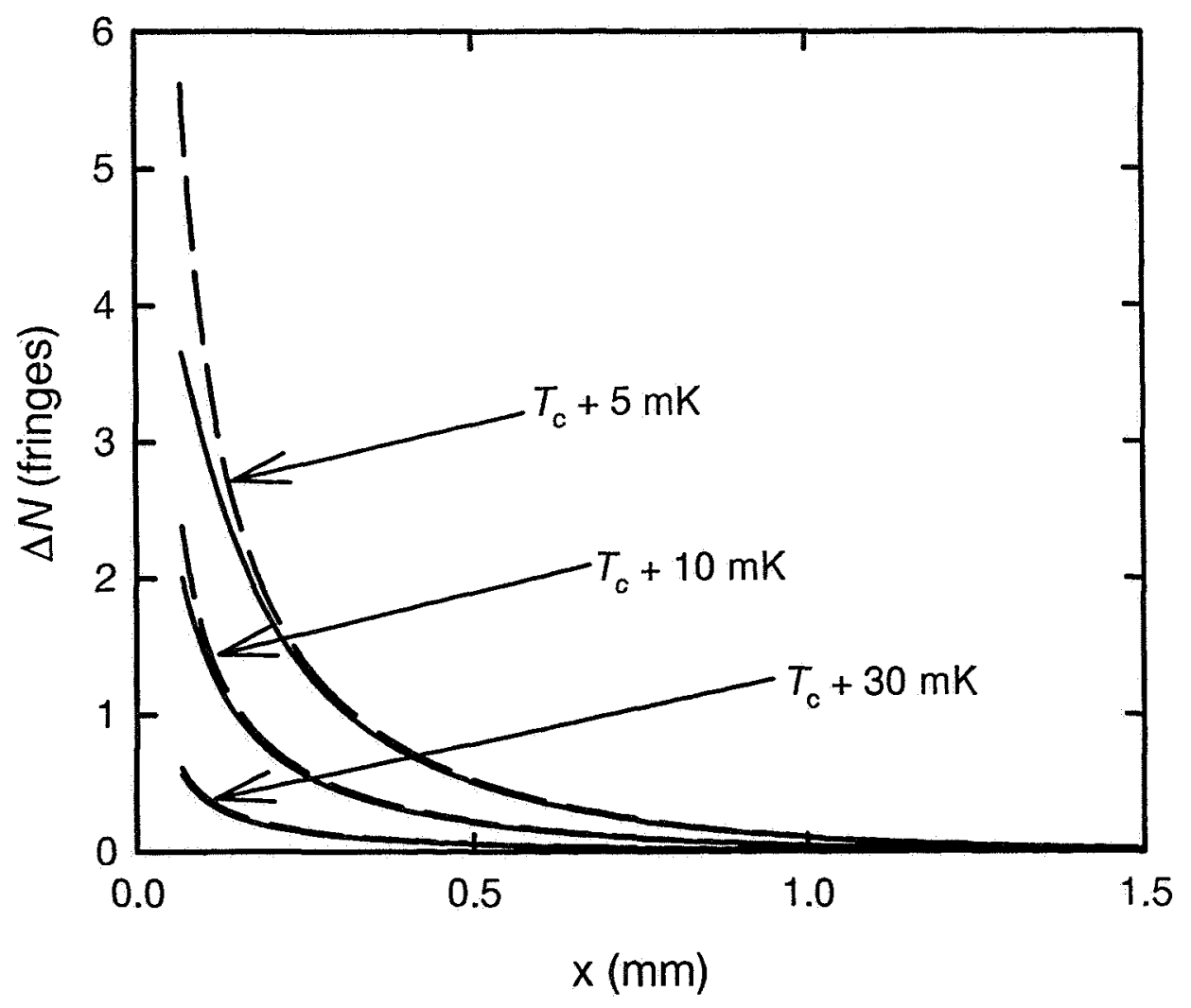

Figure 4 


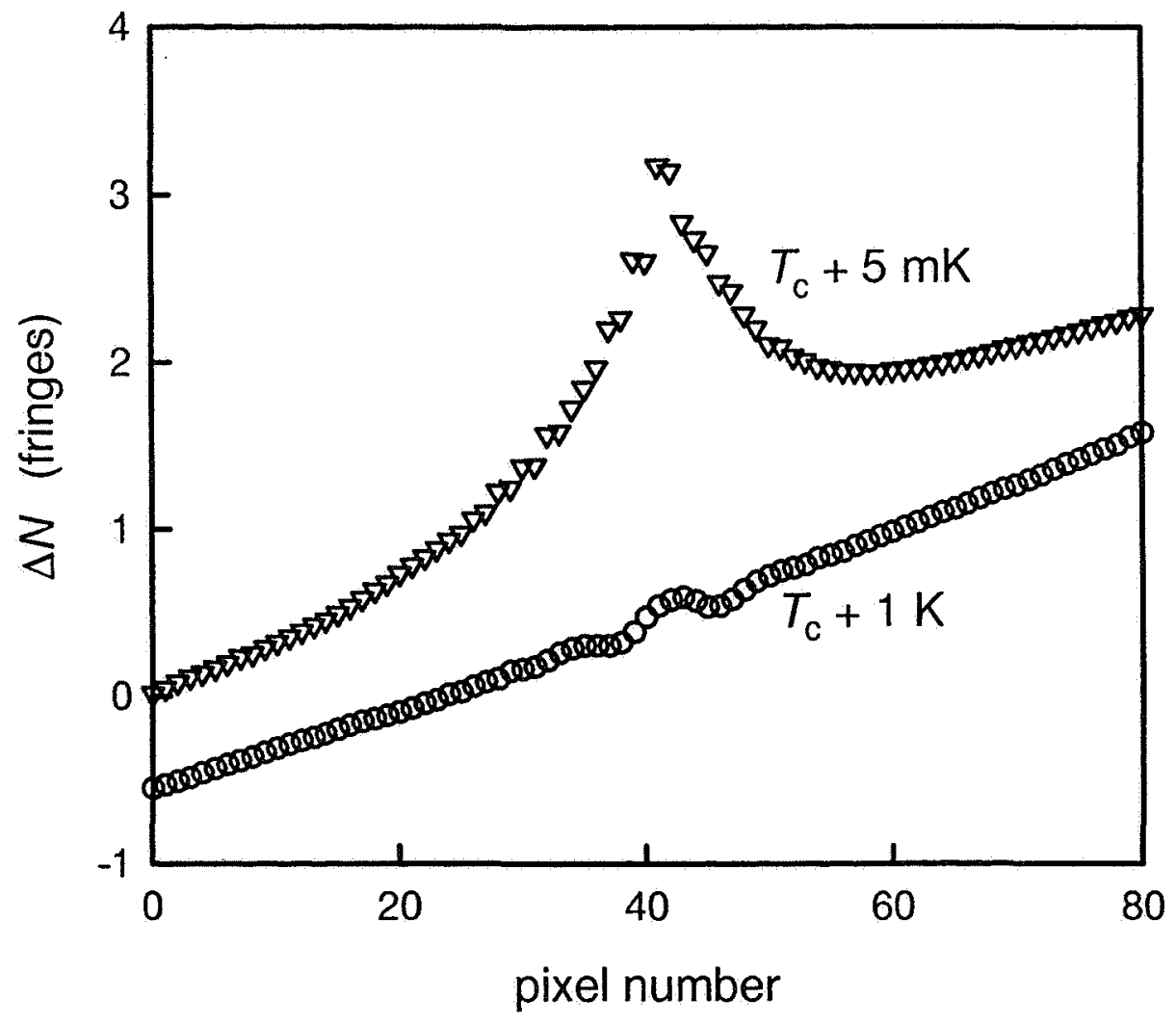

Figure 5 


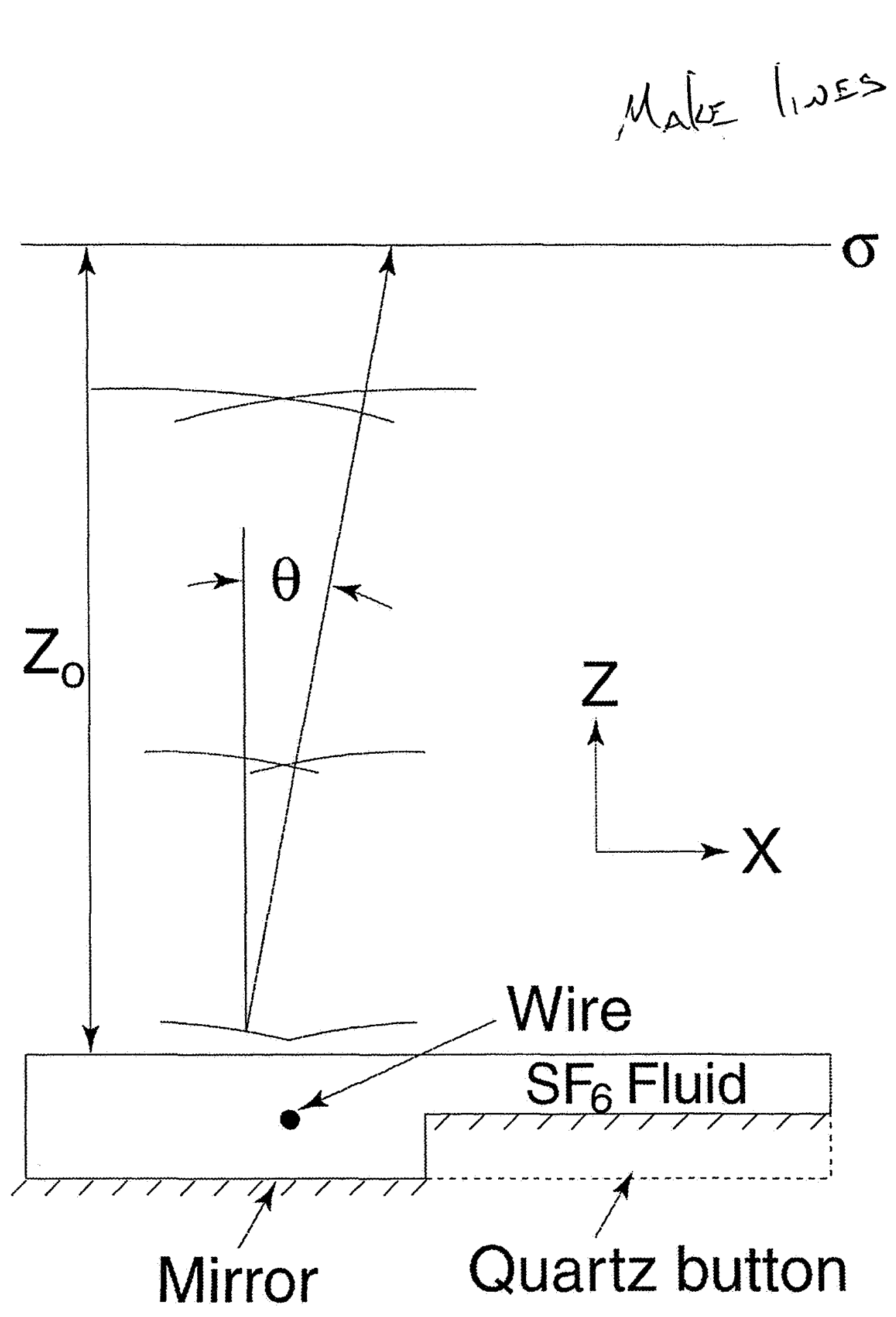

Figure 6 


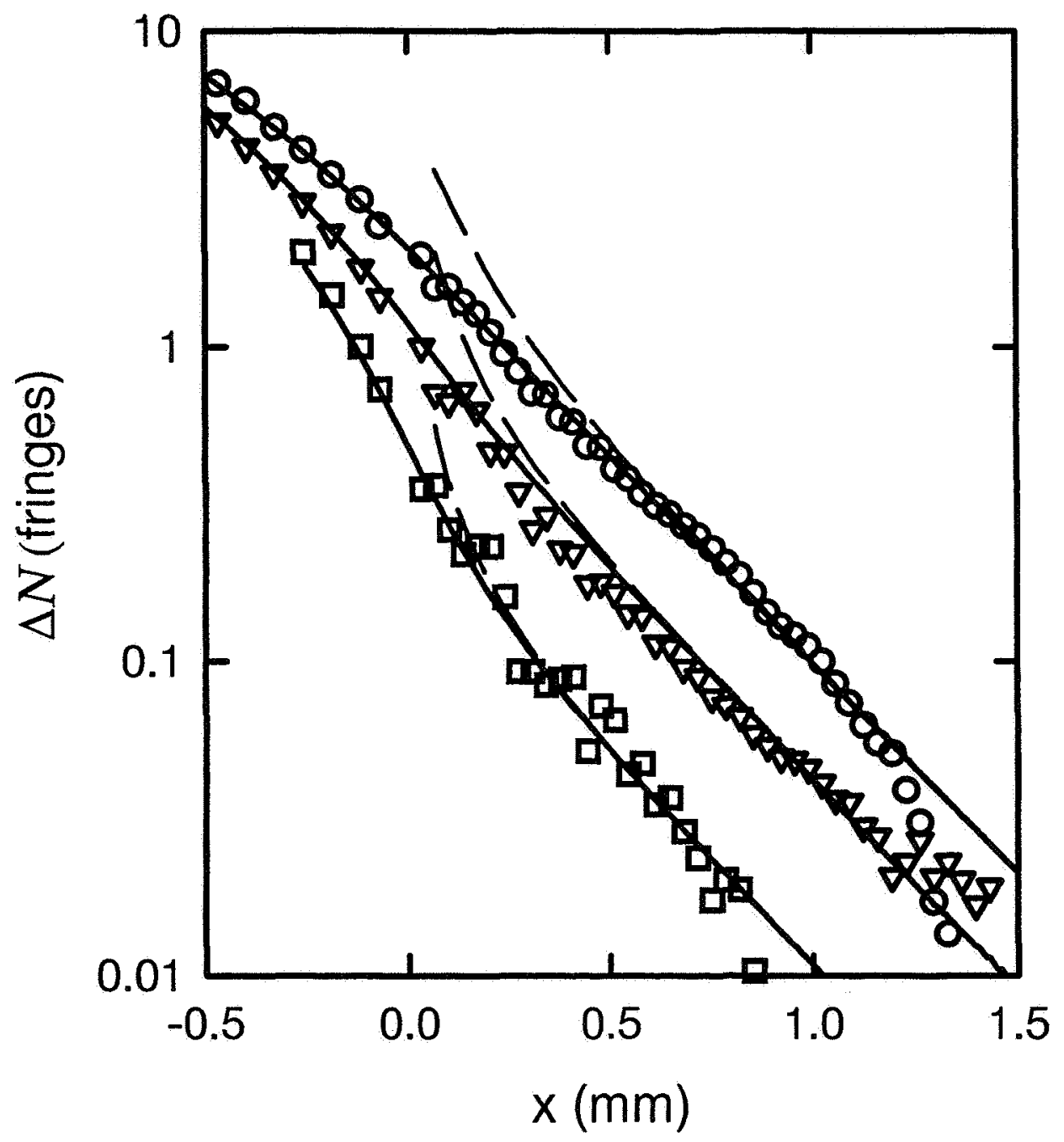

Figure 7 